University of Nebraska - Lincoln

DigitalCommons@University of Nebraska - Lincoln

U.S. Department of Veterans Affairs Staff

Publications

U.S. Department of Veterans Affairs

2007

\title{
Early life stress and novelty seeking behavior in adolescent monkeys
}

Karen J. Parker

Stanford University School of Medicine, kjparker@stanford.edu

Kimberly L. Rainwater

Stanford University School of Medicine

Christine L. Buckmaster

Stanford University School of Medicine

Alan F. Schatzberg

Stanford University School of Medicine, afschatz@stanford.edu

Steven E. Lindley

Stanford University School of Medicine, lindleys@stanford.edu

See next page for additional authors

Follow this and additional works at: https://digitalcommons.unl.edu/veterans

Parker, Karen J.; Rainwater, Kimberly L.; Buckmaster, Christine L.; Schatzberg, Alan F.; Lindley, Steven E.; and Lyons, David M., "Early life stress and novelty seeking behavior in adolescent monkeys" (2007). U.S. Department of Veterans Affairs Staff Publications. 63.

https://digitalcommons.unl.edu/veterans/63

This Article is brought to you for free and open access by the U.S. Department of Veterans Affairs at DigitalCommons@University of Nebraska - Lincoln. It has been accepted for inclusion in U.S. Department of Veterans Affairs Staff Publications by an authorized administrator of DigitalCommons@University of Nebraska - Lincoln. 


\section{Authors}

Karen J. Parker, Kimberly L. Rainwater, Christine L. Buckmaster, Alan F. Schatzberg, Steven E. Lindley, and David M. Lyons 


\title{
Early life stress and novelty seeking behavior in adolescent monkeys
}

\author{
Karen J. Parker ${ }^{a, *}$, Kimberly L. Rainwater ${ }^{a}$, Christine L. Buckmaster ${ }^{a}$, \\ Alan F. Schatzberg ${ }^{\mathrm{a}}$, Steven E. Lindley ${ }^{\mathrm{a}, \mathrm{b}}$, David M. Lyons ${ }^{\mathrm{a}}$
}

\author{
${ }^{a}$ Department of Psychiatry and Behavioral Sciences, Stanford University School of Medicine, 1201 Welch Road, \\ MSLS Room P104, Mail Code 5485, Stanford, CA 94305-5485, USA \\ ${ }^{\mathrm{b}}$ Department of Veterans Affairs, Veterans Administration Palo Alto Health Care System, Palo Alto, CA 94301-1290, USA
}

Received 1 August 2006; received in revised form 11 May 2007; accepted 16 May 2007

KEYWORDS
HPA axis;
Monkey;
Monoamines;
Novelty seeking;
Resilience;
Stress

*Corresponding author. Tel.: +1650736 9863; fax: +16504987761.

E-mail address: kjparker@stanford.edu (K.J. Parker).

\begin{abstract}
Summary
Recent evidence suggests that early exposure to mild stress promotes the development of novelty seeking behavior. Here we test this hypothesis in squirrel monkeys and investigate whether novelty seeking behavior is associated with differences in cerebrospinal fluid (CSF) levels of the serotonin metabolite 5 -hydroxyindoleacetic acid (5HIAA), the dopamine metabolite homovanillic acid (HVA), the norepinephrine metabolite 3-methoxy-4-hydroxyphenylethylene glycol (MHPG), and the neuropeptide corticotrophin-releasing factor (CRF). Monkeys were randomized early in life to either mild intermittent stress (IS) or no stress (NS) conditions, and subsequently presented with opportunities to interact with a familiar or novel object in a test box that was connected to each monkey's home cage. To further minimize the potentially stressful nature of the test situation, monkeys were acclimated to the test procedures prior to study initiation. Post-test plasma levels of cortisol in IS and NS monkeys did not differ significantly from baseline levels measured in undisturbed conditions. During testing, more IS than NS monkeys voluntarily left the home cage, and IS monkeys spent more time in the test box compared to NS monkeys. More IS than NS monkeys engaged in object exploration in the test box, and IS monkeys preferred to interact with the novel vs. familiar object. Novelty seeking was not associated with differences in 5HIAA, HVA, MHPG, or CRF, but correlated with differences in object exploration observed in a different test situation at an earlier age. These trait-like differences in novelty seeking appear to reflect mild early stress-induced adaptations that enhance curiosity and resilience.
\end{abstract}

(c) 2007 Elsevier Ltd. All rights reserved. 


\section{Introduction}

Early exposure to severe forms of stress is a risk factor for the development of subsequent psychopathology (Agid et al., 1999; Heim et al., 2004). Interestingly, however, mildly stressful early experiences appear to promote the development of resilience (Fergus and Zimmerman, 2005; Rutter, 1993). People cope better with spousal loss, work-related stressors, and major accidents if they have previously experienced and coped with stress in childhood (Forest, 1991; Khoshaba and Maddi, 1999; Mortimer and Staff, 2004). Young monkeys exposed to intermittent stress (IS) and subsequently studied in unfamiliar situations likewise show fewer distress vocalizations and decreased maternal clinging compared to monkeys raised under no stress (NS) control conditions (Levine and Mody, 2003; Lyons et al., 1999; Parker et al., 2004, 2006). IS monkeys also more quickly initiate exploration and prefer to interact with novel vs. familiar objects compared to NS monkeys (Parker et al., 2004). These differences are of interest because engagement and mastery of novel situations is thought to foster adaptations that enhance self-regulation and resilience (Kashdan et al., 2004; Reio et al., 2006).

In our previous studies, differences between IS and NS monkeys in novelty seeking were confounded with differences in plasma levels of the stress hormone cortisol. Specifically, post-test cortisol levels were higher in NS compared to IS monkeys (Parker et al., 2004). The present study was designed to determine whether novelty seeking differences persist when adrenocortical responses to the test situation are minimized. This was accomplished by extensively acclimating monkeys to, and allowing monkeys to choose between, familiar and unfamiliar elements of the test. Novelty seeking behavior observed in this test situation was then compared to novelty seeking discerned in a previous study of the same monkeys (Parker et al., 2004) to examine cross-situational consistency in the expression of this behavior. We also investigated whether novelty seeking is associated with differences in cerebrospinal fluid (CSF) measures of various neurochemicals, as studies of humans and animals have linked novelty seeking to monoamines (Bardo et al., 1996; Gerra et al., 1999; Sara et al., 1995; Zuckerman, 1985) and stress-related neuropeptides (Kabbaj et al., 2000; Thorsell et al., 2006). We therefore examined relationships between novelty seeking and CSF levels of the serotonin metabolite 5-hydroxyindoleacetic acid (5HIAA), the dopamine metabolite homovanillic acid (HVA), the norepinephrine metabolite 3-methoxy-4-hydroxyphenylethylene glycol (MHPG), and the neuropeptide corticotrophin-releasing factor (CRF).

\section{Methods}

\subsection{Subjects}

Twenty squirrel monkeys (Samiri sciureus) of Guyanese origin born at the Stanford Research Animal Facility served as subjects. All monkeys received number tags worn on necklaces to facilitate easy identification. Subjects were reared in natal groups composed of three to four motherinfant pairs. Seasonal synchronous breeding facilitated generation of an age-matched study cohort, and all infants were born within a 2-month period. Group composition was determined by infant birth dates to minimize developmental differences between infants in the same natal group. Puberty occurs between 2.5 and 3.5 years of age in squirrel monkeys, and their average maximum lifespan in captivity is $\sim 21$ years of age (Baldwin, 1985; Brady, 2000). All procedures were approved by Stanford University's Administrative Panel on Laboratory Animal Care and were carried out in accordance with the National Institutes of Health's Guide for the Care and Use of Laboratory Animals.

\subsection{Animal husbandry and rearing protocols}

Subjects were housed indoors in $1.8 \times 1.2 \times 1.8$-m wiremesh cages that were cleaned daily. Housing and testing occurred in climate-controlled rooms on a 12:12 light/dark cycle with an ambient temperature of $26^{\circ} \mathrm{C}$. Monkeys had ad libitum access to water, food (e.g., commercial New World monkey chow; fresh fruits and vegetables), and a variety of toys that provided environmental enrichment. A sliding door in each home cage facilitated access to a small, portable capture cage. Monkeys were trained to enter the capture cage on vocal command to facilitate experimental manipulations.

Subjects remained undisturbed in their natal groups through 16 weeks of age. At 17 weeks of age, natal groups were randomly assigned to one of two experimental rearing conditions. In one condition, 11 infants (seven females and four males) from four natal groups were exposed to a mild intermittent stress (IS) protocol. From weeks 17-27 of age, each infant was removed from the mother and natal group for a 1-h period once a week, placed in a cage adjacent to unfamiliar monkeys in a different room, and temporarily deprived of all forms of contact with the natal group. In the other condition, nine infants (eight females and one male) from three natal groups remained undisturbed in their home cages as NS controls.

Following completion of these rearing protocols, all monkeys were tested at 35 and 50 weeks of age for rearing differences in anxiety and neuroendocrine stress responsivity (Parker et al., 2004). Mothers were removed from the premises and returned to the breeding colony when their offspring were 1 year of age. With the exception of cognitive testing at 1.5 years of age (Parker et al., 2005), young monkeys remained in their mixed-sex peer natal groups ( $N=3-4$ monkeys per group) between 1 and 2.25 years of age. Following baseline CSF collection at 2.25 years of age (see below), monkeys were housed in larger same-sex peer groups ( $N=5-9$ monkeys per group) to preclude unplanned pregnancies. Previously closed partitions were opened between adjacent wire-mesh cages to accommodate increased group sizes. Newly formed groups were thus housed in cages composed of two $(3.6 \times 2.4 \times 3.6-\mathrm{m})$ or three $(5.4 \times 3.6 \times 5.4-\mathrm{m})$ connected compartments based on group size.

\subsection{Novelty seeking test}

Novelty seeking was assessed at 2.5 years of age in one compartment of the home cage. Because individual testing 
necessitated separation from the group, each subject was extensively acclimated to group separation for a 6 -h period once a week for 8 weeks prior to the beginning of the experiment. During acclimation (and testing), subjects had unrestricted vocal and limited visual and tactile contact with their group, and full visual and vocal contact with other monkeys in nearby cages. Monkeys were also visually exposed to the test apparatus (e.g., the novel test box and camera described below) during the final acclimation period. These procedures were designed to familiarize monkeys with, and diminish the potentially stressful nature of, the test procedure.

Assessment of novelty seeking occurred in a novel test box $(63.5 \times 50.8 \times 50.8-\mathrm{cm})$ attached to each subject's home cage. The box was composed of three opaque white acrylic plastic side panels, a clear acrylic top, concrete floor, and one open side attached at floor level to the home cage. The clear top facilitated video recording of each test session with a small digital camera (DigiCam Surveillance, LLC; Slidell, LA). Free access to the box from the home cage was provided when a sliding partition was removed from an inter-connecting opening $(30 \times 30-\mathrm{cm})$. The box contained one familiar object (i.e., a rubber ball), to which monkeys had been previously exposed for at least eight consecutive weeks in the home cage, and one unfamiliar object (i.e., an acrylic wheel). Both objects were secured to the back wall of the box, and the objects and box were cleaned between test sessions.

Monkeys spent $30 \mathrm{~min}$ alone in their home cage compartment before each test session. The box was then affixed to the compartment $5 \mathrm{~min}$ before the beginning of each test session, after which time the partition was removed and monkeys had free access to the box. Test sessions lasted 30 min in duration and each monkey was tested for five consecutive days to determine whether differences in novelty seeking are stable over time. Test sessions occurred between 1400 and $1800 \mathrm{~h}$, and testing occurred at the same time each day for a given monkey. On days when multiple subjects were tested, 40 min elapsed between the completion of one test session and the beginning of the next. Each week, 1-4 monkeys were tested and the order of testing for IS and NS monkeys was evenly distributed across daily and weekly schedules.

Video-recorded behavior was later scored by a trained observer (KLR) who was unaware of each monkey's rearing condition. The following behavior measures were analyzed for each test session: (1) latency to contact the box threshold (scored when any part of the monkey's body, excluding the tail, first contacted the threshold), (2) box threshold counts and duration (scored each time any part of the monkey's body, excluding the tail, contacted the threshold), (3) latency to enter the box (scored when the monkey's entire body, excluding the tail, first entered the box), (4) box entry counts and duration (scored each time the monkey's entire body, excluding the tail, was in the box), (5) latency to begin familiar or novel object exploration (scored when the monkey first touched each object), and (6) familiar and novel object exploration counts and duration (scored each time the monkey touched an object). Monkeys that did not enter the box or explore objects during the 30 min test session were assigned the maximal latency score of $30 \mathrm{~min}$.

\subsection{Blood sample collection and measurement}

Blood samples were collected between 1530 and $1600 \mathrm{~h}$ from all monkeys 2 weeks before the beginning of the acclimation period and 2 weeks after completion of the repeated test sessions to establish a summary measure of baseline cortisol levels in undisturbed home cage conditions. Blood samples were also collected immediately after the first and last test sessions to examine the adrenocortical response to repeated testing. Post-test blood samples were collected between 1430 and $1810 \mathrm{~h}$. Cortisol levels during this late afternoon period are relatively stable compared to a similar $3 \mathrm{~h} 40 \mathrm{~min}$ period during post-wake morning hours. Our sampling period was therefore selected to help control for fluctuations in the diurnal cortisol rhythm (Zeitzer et al., 2003). Blood samples were collected from manually restrained monkeys while blood $(1 \mathrm{ml})$ was drawn from the femoral vein with a sterile single-use syringe containing $20 \mu \mathrm{L}$ of ethylenediamine tetraacetic acid (EDTA). Blood samples were then centrifuged at $4{ }^{\circ} \mathrm{C}$ and the plasma fraction was transferred to a chilled polypropylene tube and frozen on dry ice. Most blood samples were collected within $3 \mathrm{~min}$ from cage entry (median latency to sample collection $=130 \mathrm{~s}$, range $=44-477 \mathrm{~s})$. All blood samples were stored at $-80^{\circ} \mathrm{C}$ prior to quantification. Cortisol was measured in duplicate using a commercially prepared radioimmunoassay (Diagnostic Products Corporation; Los Angeles, CA). Complete sample subsets from each condition were included in every assay. Intra- and inter-assay coefficients of variation were, respectively, $2.4 \%$ and $5.9 \%$. Sensitivity of the cortisol assay was $3 \mu \mathrm{g} / \mathrm{dl}$.

\subsection{CSF sample collection and measurement}

Baseline CSF samples were collected at 1.25 and 2.25 years of age in undisturbed home cage conditions to assess the consistency of individual differences and correlations between CSF measures and novelty seeking behavior. Samples were collected between 1500 and $1700 \mathrm{~h}$ to control for circadian variation in monoamine metabolite and CRF levels (Kalin et al., 1987; Wada et al., 1998). Anesthesia was induced by intrasaphenous injection of $10 \mathrm{mg} / \mathrm{kg}$ ketamine hydrochloride and $0.5 \mathrm{mg} / \mathrm{kg}$ diazepam, and supplemented as needed with intramuscular injection of $5 \mathrm{mg} / \mathrm{kg}$ ketamine hydrochloride. Using a sterile single-use syringe, 100-200 $\mu \mathrm{l}$ of CSF were drawn from the cistern, immediately transferred to a chilled polypropylene tube, and flash-frozen on dry ice. Although ketamine and handling stress have been shown to alter central monoamine concentrations, previous studies of rhesus and vervet monkeys have found no effect of ketamine or handling stress on CSF monoamine metabolite levels when samples were collected within $30 \mathrm{~min}$ of ketamine injection (Bacopoulos et al., 1979; Higley et al., 1991; Mehlman et al., 2000; Shelton et al., 1988). In the present experiment, all collection latencies were well within this time frame, as most CSF samples were collected within 5 min of anesthesia administration (median latency to sample collection $=190 \mathrm{~s}$, range $=60-497 \mathrm{~s}$ ) and $12 \mathrm{~min}$ from cage entry (median latency to sample collection $=562 \mathrm{~s}$, range $=308-991 \mathrm{~s}$ ). Monkeys were continuously monitored until fully recovered and then returned to 
the home cage. All CSF samples were stored at $-80^{\circ} \mathrm{C}$ prior to quantification.

CRF was measured in duplicate using a commercially prepared radioimmunoassay (Peninsula Laboratories Inc.; San Carlos, CA). All CRF samples were run in a single assay, and the intra-assay coefficient of variation was $5.8 \%$. Sensitivity of the CRF assay was $10 \mathrm{pg} / \mathrm{ml}$. Concentrations of 5HIAA, HVA, and MHPG were determined by high performance liquid chromatography (HPLC) with electrochemical detection. CSF samples were thawed, centrifuged for $2 \mathrm{~min}$, and $5 \mu \mathrm{l}$ were injected directly on a C18 reverse phase analytical column $(5 \mathrm{~mm}, 250 \times 4.6 \mathrm{~mm}$; Biophase ODS, BAS; West Lafayette, IN) protected by a precolumn cartridge $(5 \mathrm{~mm}, 30 \times 4.6 \mathrm{~mm}, \mathrm{BAS})$ and a dual analytical electrode cell set at -0.02 and $+0.35 \mathrm{~V}$ (ESA; Bedford, MA), respectively, as previously described with modification (Lindley et al., 1988). The mobile phase consisted of $0.05 \mathrm{M}$ sodium phosphate, $0.03 \mathrm{M}$ Citric Acid, $12.5 \%$ methanol, $0.03 \%$ Octyl sodium sulfate, $0.1 \mathrm{mM}$ EDTA adjusted to $\mathrm{pH}$ 2.3. Measures of CSF-derived monoamine metabolites were used to estimate the activity of their respective source neuronal systems in this experiment because intact monoamines are often undetectable in CSF. Technicians were blind to experimental conditions while conducting all radioimmunoassays and HPLC.

\subsection{Data analysis}

The effects of mild early stress exposure on novelty seeking behavior, plasma cortisol levels, and CSF monoamine and CRF levels were assessed with repeated measures analysis of variance (ANOVA). Rearing condition was considered the between-subjects factor, while test session (days 1-5), plasma assessment (baseline and post-test samples at 2.5 years of age), and CSF assessment (baseline samples at 1.25 and 2.25 years of age) were considered the repeated measures, within-subjects factors. Because this experiment was not adequately powered to detect sex differences, sex was excluded from analysis. Chi-square analysis was used to determine whether mild early stress exposure affected the proportion of monkeys that engaged in novelty seeking. Paired $t$-tests were used to examine whether monkeys more often engaged in familiar versus novel object exploration. The stability of individual differences in behavior over repeated test sessions was evaluated with Kendall's coefficient of concordance (W). Principal components analysis was then used to generate a single summary index that correlated highly with the measures assessed in the novelty-seeking test. Spearman rank order correlations $\left(r_{\mathrm{s}}\right)$ were used to examine (1) the consistency of individual differences for each repeated CSF measure, (2) the relationships between CSF measures and the summary index of novelty seeking, and (3) the relationships between the summary index of novelty seeking at 2.5 years of age and previously collected measures of novelty seeking at 35 weeks of age (Parker et al., 2004). Because individual differences in one situation can be associated with differences in another situation without the relationship being linear, rank order correlation statistics were more appropriate for these analyses than Pearson productmoment correlations. Test statistics were evaluated with two-tail probabilities $(p<0.05)$ and descriptive statistics are presented as mean \pm SEM.

\section{Results}

\subsection{Rearing differences in novelty seeking behavior}

All IS monkeys (11 of 11) and all but one of the NS monkeys (8 of 9) approached the threshold of the novel test box and peered into it during testing $\left(\chi^{2}=1.287, \mathrm{df}=19\right.$, $p=0.257)$. Monkeys from both of the rearing conditions were therefore aware of the test box, but IS monkeys contacted the box threshold faster $\left(F_{1,18}=7.425\right.$, $p=0.014)$, were in more frequent contact with the box threshold $\left(F_{1,18}=12.876, p=0.002\right)$, and spent more time on the box threshold $\left(F_{1,18}=5.584, p=0.030\right)$ compared to NS monkeys. More IS (11 of 11) than NS (5 of 9) monkeys likewise chose to enter the box $\left(\chi^{2}=6.111, \mathrm{df}=19\right.$, $p=0.013)$. IS monkeys entered faster $\left(F_{1,18}=13.124, p=\right.$ $0.002)$, did so with greater frequency $\left(F_{1,18}=9.008\right.$, $p=0.008)$, and spent more time in the box $\left(F_{1,18}=4.863\right.$, $p=0.041$ ) compared to NS monkeys.

Latencies to contact the box threshold and enter the box did not differ significantly over repeated test sessions, but rearing condition-by-test session interactions were discerned for other measures. Over the five repeated test sessions, IS monkeys less often contacted the box threshold $\left(F_{4,40}=5.834, p=0.001\right)$, spent less time on the box threshold $\left(F_{4,40}=10.447, p<0.0001\right)$, entered the box less often $\left(F_{4,40}=7.615, p<0.0001\right)$, and spent less time in the box $\left(F_{4,40}=4.013, p=0.008\right)$. These same measures for NS monkeys consistently remained at low levels (Fig. 1).

More IS (9 of 11) than NS ( 2 of 9 ) monkeys engaged in object exploration in the test box $\left(\chi^{2}=7.103, \mathrm{df}=19\right.$, $p=0.008)$. Because only two of the NS monkeys engaged in object exploration, further analysis of object exploration was restricted to IS monkeys. A significant preference for exploration of novel vs. familiar objects was discerned for the IS monkeys. Specifically, IS monkeys explored novel vs. familiar objects more frequently $(80 \pm 22$ vs. $50 \pm 17$ contact counts; $\left.t_{10}=2.280, p=0.046\right)$ and for longer durations $\left(504 \pm 156\right.$ vs. $\left.204 \pm 67 \mathrm{~s} ; t_{10}=3.012, p=0.013\right)$. Exploration latencies for novel vs. familiar objects were not significantly different. Latencies, frequencies, and durations did not differ significantly across repeated test sessions for object exploration in IS monkeys.

\subsection{Consistency of novelty seeking measures within and between test sessions}

Kendall's coefficient of concordance computed separately for six measures of behavior exhibited by IS and NS monkeys indicated that individual differences were consistent across repeated test sessions (threshold latency: $W=0.71$, $\mathrm{df}=19, p<0.0001$; threshold frequency: $W=0.71, \mathrm{df}=$ 19, $p<0.0001$; threshold duration: $W=0.80, \mathrm{df}=19$, $p<0.0001$; box entry latency: $W=0.75, \mathrm{df}=19, \quad p<0$. 0001; box entry frequency: $W=0.80, \mathrm{df}=19, p<0.0001$; box duration: $W=0.81, \mathrm{df}=19, p<0.0001)$. The mean for each measure was therefore used for the PCA to 

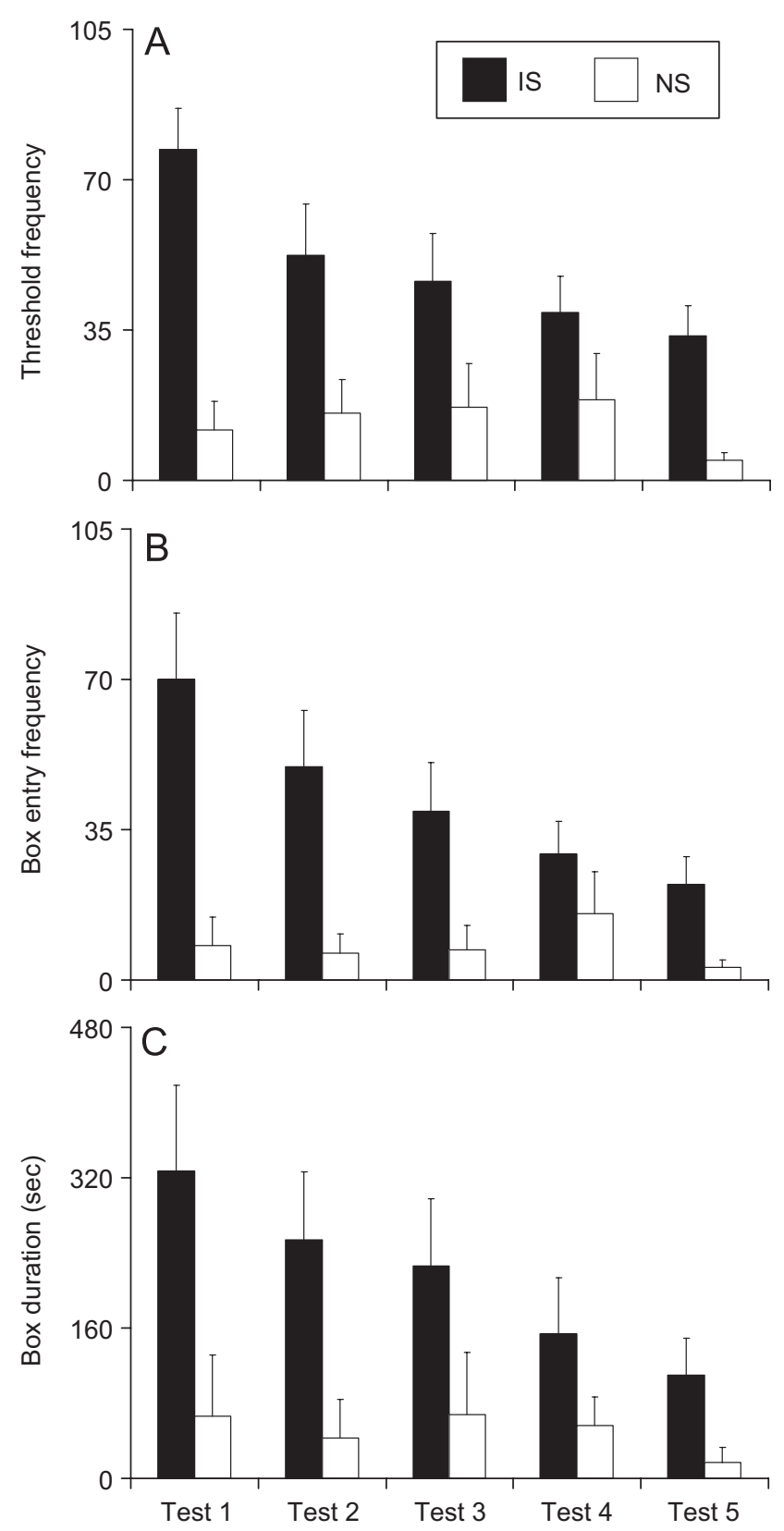

Figure 1 Rearing differences in novelty seeking at 2.5 years of age. Measures of (A) frequency to contact the novel box's threshold, (B) frequency to enter the novel box, and (C) duration spent in the novel box across the five test sessions are presented for adolescent monkeys previously exposed to either a mild intermittent stress (IS; $N=11$ ) or no stress (NS; $N=9$ ) rearing condition. Data are presented as mean \pm SEM.

generate a single summary score of novelty seeking behavior for each of the monkeys. All six measures were highly correlated with one another $\left(r_{\mathrm{s}}\right.$ range: 0.89-0.98), and all six measures loaded onto Factor 1 in the PCA which explained $87 \%$ of the total variance. Based on the following factor loadings (threshold latency $=0.84$, threshold frequency $=0.98$, threshold duration $=0.97$, box entry latency $=0.93$, box entry frequency $=0.96$, box duration $=0.92$ ), Factor 1 provided a suitable summary index of novelty seeking.

\subsection{Plasma cortisol measures}

Novelty seeking behavior occurred in the absence of adrenocortical activation in both IS and NS monkeys. Cortisol levels immediately after test sessions 1 and 5 did not differ significantly from baseline levels measured in undisturbed home cage conditions for monkeys from both rearing conditions $\left(F_{2,36}=0.847, p=0.434\right)$. This outcome was evident whether or not the time of blood sample collection was included in the statistical analysis as a covariate. Baseline cortisol levels in undisturbed home cage conditions before initiation or after completion of the novelty test sessions did not differ significantly in IS compared to NS monkeys $\left(F_{1,18}=1.012, p=0.328\right)$. Neither baseline nor post-test cortisol levels correlated with the summary index of novelty seeking behavior derived by PCA.

\subsection{CSF measures of monoamine metabolites and CRF}

CSF levels of 5HIAA, HVA, and CRF did not differ significantly in IS compared to NS monkeys (Table 1). A rearing conditionby-age interaction was discerned for CSF levels of MHPG $\left(F_{1,18}=4.527, p=0.047\right)$. IS compared to NS monkeys had lower levels of MHPG at 1.25 years of age $\left(F_{1,18}=4.731\right.$, $p=0.043$ ), but rearing differences were no longer evident at 2.25 years of age (Table 1). From 1.25 to 2.25 years of age, MHPG, 5HIAA, and HVA levels declined significantly in monkeys from both rearing conditions, while similar declines in CRF levels did not reach statistical significance (Table 1). Despite these age-related declines in monoamine metabolite levels, the relative rank order of individual differences was consistent across repeated sampling points for CSF levels of 5 HIAA, HVA, and CRF, but not MHPG (Table 1). None of the CSF measures correlated with the summary noveltyseeking index derived by PCA.

\subsection{Consistency of individual differences in behavior across situations}

In an earlier experiment, at 35 weeks of age, novelty seeking behavior was assessed in the same monkeys under inescapable conditions that involved involuntary separation from the home cage and occurred in the presence of each monkey's mother (Parker et al., 2004). The relative rank order of individual differences in novelty seeking in the earlier experiment and the summary index of novelty seeking behavior described above was remarkably consistent. Specifically, the summary novelty seeking index derived by PCA at 2.5 years of age correlated with object exploration latencies $\left(r_{\mathrm{s}}=-0.479, \mathrm{df}=18, p<0.05\right)$ and novel object exploration frequencies $\left(r_{\mathrm{s}}=0.491, \mathrm{df}=18\right.$, $p<0.05$ ) at 35 weeks of age.

\section{Discussion}

Results from this study confirm that IS monkeys engage in more novelty seeking behavior than NS monkeys. Differences in novelty seeking occurred in the absence of adrenocortical activation and do not appear to reflect 
Table 1 Rearing differences, age effects, and individual consistency in cerebrospinal fluid (CSF) monoamine metabolite and corticotropin-releasing factor (CRF) measures.

\begin{tabular}{|c|c|c|c|c|c|c|c|c|}
\hline CSF measure & Group & Year 1 & Year 2 & Age effect & $F$ & $p$ & Year 1 and 2 correlation $\left(r_{\mathrm{s}}\right)$ & $p$ \\
\hline 5HIAA (ng/ml) & $\begin{array}{l}\text { IS } \\
\text { NS }\end{array}$ & $\begin{array}{l}749 \pm 56 \\
732 \pm 63\end{array}$ & $\begin{array}{l}647 \pm 41 \\
597 \pm 48\end{array}$ & $\downarrow$ & 56.13 & $<0.0001$ & 0.90 & $<0.05$ \\
\hline HVA (ng/ml) & $\begin{array}{l}\text { IS } \\
\text { NS }\end{array}$ & $\begin{array}{l}317 \pm 15 \\
312 \pm 11\end{array}$ & $\begin{array}{l}260 \pm 11 \\
234 \pm 9\end{array}$ & $\downarrow$ & 97.86 & $<0.0001$ & 0.55 & $<0.05$ \\
\hline MHPG (ng/ml) & $\begin{array}{l}\text { IS } \\
\text { NS }\end{array}$ & $\begin{array}{l}36 \pm 2^{a} \\
44 \pm 3^{b}\end{array}$ & $\begin{array}{l}33 \pm 1 \\
32 \pm 1\end{array}$ & $\downarrow$ & 15.07 & 0.001 & -0.09 & NS \\
\hline CRF (pg/ml) & $\begin{array}{l}\text { IS } \\
\text { NS }\end{array}$ & $\begin{array}{l}108 \pm 9 \\
103 \pm 9\end{array}$ & $\begin{array}{l}98 \pm 12 \\
87 \pm 11\end{array}$ & $\leftrightarrow$ & 3.94 & 0.063 & 0.53 & $<0.05$ \\
\hline
\end{tabular}

CSF levels of 5-hydroxyindoleacetic acid (5HIAA), homovanillic acid (HVA), 3-methoxy-4-hydroxyphenylethylene glycol (MHPG), and CRF were collected at 1.25 and 2.25 years of age from adolescent monkeys previously exposed to either a mild intermittent stress (IS; $N=11$ ) or no stress (NS; $N=9$ ) rearing condition. Data are presented as mean \pm SEM. The letters "a" and "b" indicate a significant rearing difference $(p<0.05)$ within a given CSF measure. The arrows $\downarrow$ and $\leftrightarrow$ indicate, respectively, a decrease or no change in the concentration of each CSF measure between assessment points. Spearman rank order correlations $\left(r_{\mathrm{s}}\right)$ of within individual consistency for each CSF measure across assessment points are presented for all adolescent monkeys $(N=20)$.

stress-related anxiety. Novelty seeking was not associated with differences in 5HIAA, HVA, MHPG, or CRF, but correlated with differences in object exploration observed in a different test situation at an earlier age. These findings suggest that mildly stressful early experiences promote the development of trait-like differences in novelty seeking throughout adolescence when this behavior generally reaches peak levels (Arnett, 1992; Kelley et al., 2004).

Initially, all IS and NS monkeys were raised in undisturbed groups each composed of 3 or 4 mother-infant pairs. From 17 to 27 weeks of age, NS monkeys remained undisturbed in their natal groups, while IS monkeys were exposed to ten weekly $1 \mathrm{~h}$ social separations. The IS condition was designed to provide repeated opportunities for emotional activation and cognitive processing while not overwhelming the capacity for coping with stress. Separations were scheduled at weekly intervals to allow ample time for recovery at an age when squirrel monkeys are typically developing physical and psychosocial independence (Boinski and Fragaszy, 1989; Rosenblum, 1968). The transition from filial, motherdirected behavior (e.g., clinging and nursing) to exploratory, other-directed behavior (e.g., object exploration and social play) presents important learning opportunities that are thought to promote the development of various functions (Lyons, 1993; Mason, 1971). At 35 weeks of age, each IS and NS monkey was tested along with its mother for 30 min on five consecutive days in a novel environment that contained an assortment of foods and toy-like objects (Parker et al., 2004). Similar test conditions have been used to demonstrate that prior exposure to overwhelming postnatal stressors decreases novelty seeking and increases indications of anxiety in marmosets and macaques (Andrews and Rosenblum, 1993; Dettling et al., 2002; Hinde and SpencerBooth, 1971; Roder et al., 1989; Sackett, 1972). In contrast, we found that IS monkeys are less anxious in this test situation as inferred by decreased maternal clinging and increased object exploration compared to NS monkeys (Parker et al., 2004). Similar findings were previously reported for a different group of IS monkeys that responded to the removal of mothers at weaning with fewer distress vocalizations and more time spent near peers (Lyons et al., 1999). In our previous studies, differences between novelty seeking were confounded with differences in plasma levels of cortisol. Here we show that early experience-dependent differences in novelty seeking persist in the absence of rearing differences in plasma levels of cortisol and CSF levels of 5HIAA, HVA, MHPG, and CRF.

Monoamine metabolite levels in repeated samples of CSF declined with age as reported for human children and macaque monkeys (Higley et al., 1992; Komori et al., 1999; Langlais et al., 1985; Shelton et al., 1988). Age-related declines are thought to reflect diminished monoamine synthesis and, to a lesser extent, increased degradation (Komori et al., 1999; Nicotra et al., 2004; Rind et al., 2000). We also observed interindividual temporal stability in CSF levels of $5 \mathrm{HIAA}, \mathrm{HVA}$, and CRF. Interindividual stability was not observed for CSF levels of MHPG or plasma levels of cortisol. Previous studies of vervet and macaque monkeys suggest that CSF levels of 5HIAA and HVA exhibit temporal stability across a variety of circumstances (Higley et al., 1992; Raleigh et al., 1992). CSF levels of norepinephrine and plasma levels of cortisol show temporal and situational stability only in the absence of early life stress exposure (Capitanio et al., 1998; Higley et al., 1996). These findings indicate that 5HIAA and HVA measures may be less sensitive to early experience-dependent changes than are CSF levels of MHPG or plasma levels of cortisol.

In addition to age-related effects, a transient early experience-dependent effect was discerned in CSF levels of MHPG, but not 5HIAA, HVA, or CRF. CSF levels of MHPG were significantly lower in IS compared to NS monkeys at 1.25 years of age, whereas differences were no longer evident at 2.25 years of age. These findings clearly require replication, but are of interest because the same IS compared to NS monkeys also had diminished baseline levels of cortisol in home cage conditions at 35 weeks and 1.5 years of age (Parker et al., 2004, 2005). Rearing differences were no longer evident for plasma levels of cortisol at 2.5 years of 
age, or CSF levels of MHPG at 2.25 years of age. Nevertheless, transient differences in MHPG or cortisol levels during early development may have lasting organizational effects that potentially lead to differences in subsequent brain functions and behavior (Becker et al., 2002).

As with all studies, several possible limitations should be considered. First, we did not find evidence for associations between novelty seeking and CSF measures used to estimate the activity of source monoamine neuronal systems in the brain. Although CSF levels of monoamine metabolites are strongly associated with their respective levels in certain brain regions (Wester et al., 1990), not all regions are wellrepresented by CSF measures. CSF measures may therefore reflect the overall activity of monoamines, but meaningful inferences are of course limited for specific brain regions. Second, the generalizability of our findings is constrained by a skewed sex ratio with more females than males, and additional research is needed to assess the effects of mild early stress on sex differences in novelty seeking. Third, our measures may reflect impulsivity and not just novelty seeking since these aspects of behavior are often associated with one another (Kelley et al., 2004; Zuckerman, 1985). This seems unlikely because IS monkeys perform significantly better than NS monkeys on a cognitive task that requires inhibition of a prepotent response to obtain highly preferred food (Parker et al., 2005). Additional studies of a similar sort are needed to establish that early experience-dependent differences in novelty seeking do not reflect differences in behavioral impulsivity.

In summary, we found that mildly stressful early experiences subsequently lead to increased novelty seeking behavior in adolescent squirrel monkeys. Exposure to mild early life stress likewise strengthens emotional and neuroendocrine resistance to subsequent stressors, and enhances performance on a challenging cognitive test (Levine and Mody, 2003; Lyons et al., 1999; Parker et al., 2004, 2006). Information-seeking and sensory types of curiosity motivate individuals to seek new opportunities for action (Reio et al., 2006). Engagement in new situations that are challenging but not overwhelming appear to foster adaptations that enhance self-regulation and resilience (Kashdan et al., 2004). From this perspective, enduring curiosity may contribute to the life-long maintenance of differences in behavior induced by exposure to early mild stress.

\section{Role of funding source}

The funding sources had no role in the collection, analysis and interpretation of data; in the writing of the report; or in the decision to submit the manuscript for publication.

\section{Conflict of interest}

All authors declare that no conflict of interest, financial or otherwise, exists with regard to this research.

\section{Acknowledgements}

Dr. Parker was supported by MH66537 from the National Institute of Mental Health, Bethesda, MD and a Stanford
University School of Medicine Dean's Fellowship. This research was supported by MH47573 and MH50604 from the National Institute of Mental Health; DA16902 from the National Institute on Drug Abuse, Bethesda, MD; and the Nancy Pritzker Network, New York, NY. We gratefully acknowledge Dr. James Ritchie for technical advice on the CRF assay, Dr. Jamie Zeitzer for assistance with blood sampling, Xiaohong She for assistance with HPLC, and Mr. Stuart Anhorn for excellent care of our animals. All authors declare that no conflict of interest, financial or otherwise, exists with regard to this research.

\section{References}

Agid, O., Shapira, B., Zislin, J., Ritsner, M., Hanin, B., Murad, H., et al., 1999. Environment and vulnerability to major psychiatric illness: a case control study of early parental loss in major depression, bipolar disorder and schizophrenia. Mol. Psychiatry 4, 163-172.

Andrews, M.W., Rosenblum, L.A., 1993. Assessment of attachment in differentially reared infant monkeys (Macaca radiata): response to separation and a novel environment. J. Comp. Psychol. 107, 84-90.

Arnett, J., 1992. Reckless behavior in adolescence: a developmental perspective. Dev. Rev. 12, 339-373.

Bacopoulos, N.G., Redmond, D.E., Roth, R.H., 1979. Serotonin and dopamine metabolites in brain regions and cerebrospinal fluid of a primate species: effects of ketamine and fluphenazine. J. Neurochem. 32, 1215-1218.

Baldwin, J.D., 1985. The behavior of squirrel monkeys (Saimiri) in natural environments. In: Rosenblum, L.A., Coe, C.L. (Eds.), Handbook of Squirrel Monkey Research. Plenum Press, New York, pp. 35-53.

Bardo, M.T., Donohew, R.L., Harrington, N.G., 1996. Psychobiology of novelty seeking and drug seeking behavior. Behav. Brain Res. 77, 23-43.

Becker, J.B., Breedlove, S.M., Crews, D., McCarthy, M.M., 2002. Behavioral Endocrinology, second ed. The MIT Press, Cambridge, MA.

Boinski, S., Fragaszy, D.M., 1989. The ontogeny of foraging in squirrel monkeys, Saimiri oerstedi. Anim. Behav. 37, 415-428.

Brady, A.G., 2000. Research techniques for the squirrel monkey (Saimiri sp.). Ilar. J. 41, 10-18.

Capitanio, J.P., Mendoza, S.P., Lerche, N.W., 1998. Individual differences in peripheral blood immunological and hormonal measures in adult male rhesus macaques (Macaca mulatta): evidence for temporal and situational consistency. Am. J. Primatol. 44, 29-41.

Dettling, A.C., Feldon, J., Pryce, C.R., 2002. Repeated parental deprivation in the infant common marmoset (Callithrix jacchus, primates) and analysis of its effects on early development. Biol. Psychiatry 52, 1037-1046.

Fergus, S., Zimmerman, M.A., 2005. Adolescent resilience: a framework for understanding healthy development in the face of risk. Annu. Rev. Public Health 26, 399-419.

Forest, K.B., 1991. The interplay of childhood stress and adult life events on women's symptoms of depression. Diss. Abstr. Int. 51, 3237.

Gerra, G., Avanzini, P., Zaimovic, A., Sartori, R., Bocchi, C., Timpano, M., et al., 1999. Neurotransmitters, neuroendocrine correlates of sensation-seeking temperament in normal humans. Neuropsychobiology 39, 207-213.

Heim, C., Plotsky, P.M., Nemeroff, C.B., 2004. Importance of studying the contributions of early adverse experience to neurobiological findings in depression. Neuropsychopharmacology 29, 641-648. 
Higley, J.D., Suomi, S.J., Linnoila, M., 1991. CSF monoamine metabolite concentrations vary according to age, rearing, and sex, and are influenced by the stressor of social separation in rhesus monkeys. Psychopharmacology (Berl.) 103, $551-556$.

Higley, J.D., Suomi, S.J., Linnoila, M., 1992. A longitudinal assessment of CSF monoamine metabolite and plasma cortisol concentrations in young rhesus monkeys. Biol. Psychiatry 32, 127-145.

Higley, J.D., King Jr., S.T., Hasert, M.F., Champoux, M., Suomi, S.J., Linnoila, M., 1996. Stability of interindividual differences in serotonin function and its relationship to severe aggression and competent social behavior in rhesus macaque females. Neuropsychopharmacology 14, 67-76.

Hinde, R.A., Spencer-Booth, Y., 1971. Effects of brief separation from mother on rhesus monkeys. Science 173, 111-118.

Kabbaj, M., Devine, D.P., Savage, V.R., Akil, H., 2000. Neurobiological correlates of individual differences in novelty-seeking behavior in the rat: differential expression of stress-related molecules. J. Neurosci. 20, 6983-6988.

Kalin, N.H., Shelton, S.E., Barksdale, C.M., Brownfield, M.S., 1987. A diurnal rhythm in cerebrospinal fluid corticotrophin-releasing hormone different from the rhythm of pituitary-adrenal activity. Brain Res. 426, 385-391.

Kashdan, T.B., Rose, P., Fincham, F.D., 2004. Curiosity and exploration: facilitating positive subjective experiences and personal growth opportunities. J. Pers. Assess. 82, 291-305.

Kelley, A.E., Schochet, T., Landry, C.F., 2004. Risk taking and novelty seeking in adolescence: introduction to part I. Ann. N Y Acad. Sci. 1021, 27-32.

Khoshaba, D.M., Maddi, S.R., 1999. Early experiences in hardiness development. Consult. Psychol. J.: Practice Res. 51, 106-116.

Komori, H., Matsuishi, T., Yamada, S., Ueda, N., Yamashita, Y., Kato, H., 1999. Effect of age on cerebrospinal fluid levels of metabolites of biopterin and biogenic amines. Acta Paediatr. 88, 1344-1347.

Langlais, P.J., Walsh, F.X., Bird, E.D., Levy, H.L., 1985. Cerebrospinal fluid neurotransmitter metabolites in neurologically normal infants and children. Pediatrics 75, 580-586.

Levine, S., Mody, T., 2003. The long-term psychobiological consequences of intermittent postnatal separation in the squirrel monkey. Neurosci. Biobehav. Rev. 27, 83-89.

Lindley, S.E., Gunnet, J.W., Lookingland, K.J., Moore, K.E., 1988. Effects of alterations in the activity of tuberohypophysial dopaminergic neurons on the secretion of alphamelanocyte stimulating hormone. Proc. Soc. Exp. Biol. Med. 188, 282-286.

Lyons, D.M., 1993. Conflict as a constructive force in social life. In: Mason, W.A., Mendoza, S.P. (Eds.), Primate Social Conflict. State University of New York Press, Albany, NY, pp. 387-408.

Lyons, D.M., Martel, F.L., Levine, S., Risch, N.J., Schatzberg, A.F., 1999. Postnatal experiences and genetic effects on squirrel monkey social affinities and emotional distress. Horm. Behav. 36, 266-275.

Mason, W.A. (Ed.), 1971. Motivational Factors in Psychosocial Development. University of Nebraska Press, Lincoln.

Mehlman, P.T., Westergaard, G.C., Hoos, B.J., Sallee, F.R., Marsh, S., Suomi, S.J., et al., 2000. CSF 5-HIAA and nighttime activity in free-ranging primates. Neuropsychopharmacology 22, 210-218.

Mortimer, J.T., Staff, J., 2004. Early work as a source of developmental discontinuity during the transition to adulthood. Dev. Psychopathol. 16, 1047-1070.
Nicotra, A., Pierucci, F., Parvez, H., Senatori, O., 2004. Monoamine oxidase expression during development and aging. Neurotoxicology 25, 155-165.

Parker, K.J., Buckmaster, C.L., Schatzberg, A.F., Lyons, D.M., 2004. Prospective investigation of stress inoculation in young monkeys. Arch. Gen. Psychiatry 61, 933-941.

Parker, K.J., Buckmaster, C.L., Justus, K.R., Schatzberg, A.F., Lyons, D.M., 2005. Mild early life stress enhances prefrontal-dependent response inhibition in monkeys. Biol. Psychiatry 57, 848-855.

Parker, K.J., Buckmaster, C.L., Sundlass, K., Schatzberg, A.F., Lyons, D.M., 2006. Maternal mediation, stress inoculation, and the development of neuroendocrine stress resistance in primates. Proc. Nat. Acad. Sci. USA 103, 3000-3005.

Raleigh, M.J., Brammer, G.L., McGuire, M.T., Pollack, D.B., Yuwiler, A., 1992. Individual differences in basal cisternal cerebrospinal fluid 5-HIAA and HVA in monkeys. The effects of gender, age, physical characteristics, and matrilineal influences. Neuropsychopharmacology 7, 295-304.

Reio Jr., T.G., Petrosko, J.M., Wiswell, A.K., Thongsukmag, J., 2006. The measurement and conceptualization of curiosity. J. Genet. Psychol. 167, 117-135.

Rind, H.B., Russo, A.F., Whittemore, S.R., 2000. Developmental regulation of tryptophan hydroxylase messenger RNA expression and enzyme activity in the raphe and its target fields. Neuroscience 101, 665-677.

Roder, E.L., Timmermans, P.J., Vossen, J.M., 1989. Effects of rearing and exposure condition upon the acquisition of phobic behaviour in cynomolgus monkeys. Behav. Res. Ther. 27, 221-231.

Rosenblum, L.A., 1968. Mother-infant relations and early behavioral development in the squirrel monkey. In: Rosenblum, L.A., Cooper, R.W. (Eds.), The Squirrel Monkey. Academic Press, New York, pp. 207-233.

Rutter, M., 1993. Resilience: some conceptual considerations. J. Adolesc. Health 14, 626-631, 690-626.

Sackett, G., 1972. Exploratory behavior of rhesus monkeys as a function of rearing experiences and sex. Dev. Psychol. 6, 260-270.

Sara, S.J., Dyon-Laurent, C., Herve, A., 1995. Novelty seeking behavior in the rat is dependent upon the integrity of the noradrenergic system. Brain Res. Cogn. Brain Res. 2, 181-187.

Shelton, S.E., Kalin, N.H., Gluck, J.P., Keresztury, M.F., Schneider, V.A., Lewis, M.H., 1988. Effect of age on cisternal cerebrospinal fluid concentrations of monoamine metabolites in nonhuman primates. Neurochem. Int. 13, 353-357.

Thorsell, A., Slawecki, C.J., El Khoury, A., Mathe, A.A., Ehlers, C.L., 2006. The effects of social isolation on neuropeptide $Y$ levels, exploratory and anxiety-related behaviors in rats. Pharmacol. Biochem. Behav. 83, 28-34.

Wada, Y., Egashira, T., Takayama, F., Yamanaka, Y., Takada, K., Takeda, H., Matsumiya, T., 1998. Variation of monoamines and their metabolite contents in the cerebrospinal fluid of conscious rats. Jpn J. Pharmacol. 78, 237-240.

Wester, P., Bergstrom, U., Eriksson, A., Gezelius, C., Hardy, J., Winblad, B., 1990. Ventricular cerebrospinal fluid monoamine transmitter and metabolite concentrations reflect human brain neurochemistry in autopsy cases. J. Neurochem. 54, 1148-1156.

Zeitzer, J.M., Buckmaster, C.L., Parker, K.J., Hauck, C.M., Lyons, D.M., Mignot, E., 2003. Circadian and homeostatic regulation of hypocretin in a primate model: implications for the consolidation of wakefulness. J. Neurosci. 23, 3555-3560.

Zuckerman, M., 1985. Sensation seeking, mania, and monoamines. Neuropsychobiology 13, 121-128. 